\title{
Altered gut microbiota profile in common variable immunodeficiency associates with levels of lipopolysaccharide and markers of systemic immune activation
}

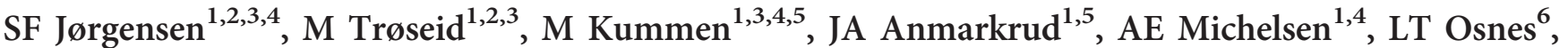 \\ K Holm $^{1,5}$, ML Høivik ${ }^{7}$, A Rashidi ${ }^{1}$, CP Dahl ${ }^{1,8}$, M Vesterhus ${ }^{5,9}$, B Halvorsen ${ }^{1,3,4}$, TE Mollnes ${ }^{3,6,10,11}$, \\ RK Berge ${ }^{12,13}$, B Moum ${ }^{4,7}$, KEA Lundin ${ }^{4,14}$, B Fevang ${ }^{1,2,4}$, T Ueland ${ }^{3,4,14,15}$, TH Karlsen ${ }^{1,3,4,5,14,16}$, \\ P Aukrust ${ }^{1,2,3,4}$ and JR Hov ${ }^{1,3,4,5,14}$
}

Common variable immunodeficiency (CVID) is the most common symptomatic primary immunodeficiency characterized by low immunoglobulin (Ig) $G$ and $\lg A$, and/or $\operatorname{lgM}$. In addition to bacterial infections, a large subgroup has noninfectious inflammatory and autoimmune complications. We performed $16 \mathrm{~S}$ ribosomal RNA-based profiling of stool samples in 44 CVID patients, 45 patients with inflammatory bowel disease (disease controls), and 263 healthy controls. We measured plasma lipopolysaccharide (LPS) and markers of immune cell activation (i.e., soluble (s) CD14 and sCD25) in an expanded cohort of 104 patients with CVID and in 30 healthy controls. We found a large shift in the microbiota of CVID patients characterized by a reduced within-individual bacterial diversity (alpha diversity, $P<0.001$ ) without obvious associations to antibiotics use. Plasma levels of both LPS $(P=0.001)$ and SCD25 $(P<0.0001)$ were elevated in CVID, correlating negatively with alpha diversity and positively with a dysbiosis index calculated from the taxonomic profile. Low alpha diversity and high dysbiosis index, LPS, and immune markers were most pronounced in the subgroup with inflammatory and autoimmune complications. Low level of IgA was associated with decreased alpha diversity, but not independently from sCD25 and LPS. Our findings suggest a link between immunodeficiency, systemic immune activation, LPS, and altered gut microbiota.

\section{INTRODUCTION}

Common variable immunodeficiency (CVID) is the most common symptomatic primary immunodeficiency disease characterized by a B-cell defect resulting in inadequate antibody responses with decreased levels of immunoglobulin (Ig)G and IgA, and/or IgM. Other immunological abnormalities include T-cell dysfunction and low-grade systemic inflammation contributing to the clinical manifestations and, potentially, B-cell dysfunction, in these patients. ${ }^{1-4}$ In addition to respiratory tract infections, a substantial proportion

\footnotetext{
${ }^{1}$ Research Institute of Internal Medicine, Division of Cancer Medicine, Surgery and Transplantation, Oslo University Hospital, Rikshospitalet, Oslo, Norway. ${ }^{2}$ Department of Transplantation Medicine, Section of Clinical Immunology and Infectious Diseases, Oslo University Hospital Rikshospitalet, Oslo, Norway. ${ }^{3} \mathrm{~K}$ G Jebsen Inflammation Research Centre, Institute of Clinical Medicine, University of Oslo, Oslo, Norway. ${ }^{4}$ Institute of Clinical Medicine, Faculty of Medicine, University of Oslo, Oslo, Norway. ${ }^{5}$ Norwegian PSC Research Center, Department of Transplantation Medicine, Oslo University Hospital Rikshospitalet, Oslo, Norway. ${ }^{6}$ Department of Immunology, Oslo University Hospital, Rikshospitalet, Oslo, Norway. ${ }^{7}$ Department of Gastroenterology, Oslo University Hospital Ullevål, Oslo, Norway. ${ }^{8}$ Department of Cardiology, Oslo University Hospital, Oslo, Norway. ${ }^{9}$ Department of Medicine, National Centre for Ultrasound in Gastroenterology, Haukeland University Hospital, Bergen, Norway. ${ }^{10}$ Research Laboratory, Nordland Hospital, Bodø, and Faculty of Health Sciences, K.G. Jebsen TREC, University of Tromsø, Tromsø, Norway. ${ }^{11}$ Centre of Molecular Inflammation Research, Norwegian University of Science and Technology, Trondheim, Norway. ${ }^{12}$ Department of Clinical Science, University of Bergen, Bergen, Norway. ${ }^{13}$ Department of Heart Disease, Haukeland University Hospital, Bergen, Norway. ${ }^{14}$ Department of Transplantation Medicine, Section of Gastroenterology, Oslo University Hospital Rikshospitalet, Oslo, Norway. ${ }^{15} \mathrm{~K}$ G Jebsen Thrombosis Research and Expertise Centre, University of Troms $\varnothing$, Troms $\varnothing$, Norway and ${ }^{16}$ Department of clinical medicine, University of Bergen, Bergen, Norway. Correspondence: SF Jørgensen or JR Hov (s.f.jorgensen@medisin.uio.no or j.e.r.hov@medisin.uio.no)
} 
Table 1 Overview of the characteristics for CVID, controls and IBD

\begin{tabular}{|c|c|c|c|c|c|}
\hline & CVID $n=44$ & Controls $n=263$ & IBD $n=45$ & $P$-value all phenotypes & $P$-value CVID-healthy \\
\hline Age in years & $50 \pm 16$ & $46 \pm 7$ & $41 \pm 14$ & $0.002^{a}$ & $0.03^{b}$ \\
\hline mean $+/-$ s.d. (range) & (18-82) & $(30-61)$ & (22-69) & & \\
\hline Male \% & 50 & 58 & 60 & $0.52^{\mathrm{c}}$ & $0.32^{d}$ \\
\hline Body Mass Index & $24.6 \pm 4.1$ & $26.4 \pm 4.1$ & $24.9 \pm 4.1$ & $0.002^{\mathrm{a}}$ & $0.007^{\mathrm{b}}$ \\
\hline mean $+/-$ s.d. (range) & (18.0-37.5) & (17.6-42.6) & (22.3-40.9) & & \\
\hline Smokers \% & 9.1 & 11.4 & 0 & $0.06^{\mathrm{c}}$ & $0.80^{d}$ \\
\hline (actual number) & (4) & (30) & (0) & & \\
\hline Courses of antibiotics in the last year ${ }^{\mathrm{e}}$ & $1.9 \pm 2.0$ & $0.3 \pm 0.7$ & $0.6 \pm 1.7$ & $0.0001^{a}$ & $<0.0001^{\mathrm{b}}$ \\
\hline mean $+/-$ s.d. (range) & $(0-7)$ & $(0-5)$ & $(0-10)$ & & \\
\hline Days before sample reach the freezer & $1.43 \pm 0.66$ & $1.46 \pm 0.50$ & $1.31 \pm 0.54$ & $0.05^{a}$ & $0.83^{\mathrm{b}}$ \\
\hline mean $+/-$ s.d. (range) & $(0.13-3.00)$ & $(0.67-3.00)$ & $(0.75-2.96)$ & & \\
\hline
\end{tabular}

Abbreviations: CVID, common variable immunodeficiency; IBD, inflammatory bowel disease.

aKruskal-Wallis of CVID, controls and IBD.

bMann-Whitney test for CVID and Controls.

${ }^{\circ}$ Chi-square of CVID, controls and IBD.

'Fisher's exact test for CVID and Controls.

Excluding the last month.

( $\sim 70 \%$ of the patients) $)^{5,6}$ have one or more noninfectious complications such as autoimmune disorders, enteropathy, and malignant and non-malignant lymphoid hyperplasia. ${ }^{5,7}$ Notably, CVID patients with noninfectious complications have increased mortality compared with patients with infections only. ${ }^{5,6}$

The etiology of CVID remains unclear. Monogenic traits have been identified in $<10 \%,{ }^{8}$ but the heritability is otherwise more consistent with a complex pattern of inheritance, meaning that multiple genetic and environmental risk factors interact to cause disease. While we have recently established robust genetic risk factors associated with CVID, ${ }^{9,10}$ no environmental risk factors have so far been identified.

The gut microbiota is important for the maturation of the immune system; germ-free mice fail to develop isolated lymphoid follicles and are also deficient in secretory $\operatorname{IgA}$, whereas colonization with commensal bacteria in these mice induces the intestinal IgA response and maturation of lymphoid follicles. ${ }^{11-15}$ The gut microbiota also influences metabolic phenotypes (e.g., obesity and insulin resistance), as well as, local and systemic inflammation, as seen in inflammatory bowel disease (IBD) and cardiovascular disorders, respectively. ${ }^{16-19}$

Previously, intestinal gene expression profiles from three patients with CVID have suggested that microbiota-induced changes could influence B-cell development, ${ }^{20}$ and Perreau et $a .^{21}$ recently suggested that gut microbiota-derived lipopolysaccharide (LPS) could induce T-cell pathology in CVID. Furthermore, experimental models have shown mice lacking B cells and IgA have reduced bacterial diversity. ${ }^{22}$ However, no available data exist on the gut microbiota composition of CVID patients.

Based on the involvement of the intestinal tract and lack of Igs shaping its microbial content, as well as, features of lowgrade systemic inflammation, we hypothesized that CVID patients are characterized by alteration in their gut microbiota composition that could be related to the patient phenotype. Hence, we performed a cross-sectional study of the gut microbiota in CVID patients and controls, relating these data to clinical and immunological characteristics as well as systemic LPS levels in these patients.

\section{RESULTS}

\section{Patient characteristics}

All the listed CVID patients $(n=120)$ at Section of Clinical Immunology and Infectious Diseases, Oslo University Hospital Rikshospitalet, representing the majority of CVID patients in Norway, were invited to participate in the study. Stool samples were retrieved from 67 CVID patients. Exclusion criteria for all the participants with stool samples were: antibiotics in the last month, sample older than $72 \mathrm{~h}$, colostomy, ileostomy, nasogastric feeding tube, and self-reported special diet like vegetarian, vegan, and low-carbohydrate diet. We excluded CVID patients on immunosuppressive medication (apart from low-dose prednisolone at $5 \mathrm{mg}$ or lower $(n=1)$ ), but not IBD patients (69\% were on immunosuppressive medication), as these primarily were used as an intestinal disease control assumed to have different microbiota. From the 67 CVID patients, 17 samples were excluded owing to antibiotics in the last month $(n=13)$ and immunosuppressive therapy $(n=4)$, whereas five samples were left out owing to various reasons, including possible misdiagnosis and the use of nasogastric feeding tube. After sequencing, one additional sample was excluded owing to low number of reads (below $9,000)(n=1)$. The gut microbiota profile was analyzed in 44 CVID patients, 45 IBD patients, and 263 healthy controls (Table 1).

Thirty-five (80\%) of the CVID patients belonged to the subgroup with "Complications", i.e., exhibited one or more of the features splenomegaly $(n=21)$, lymphadenopathy 
a

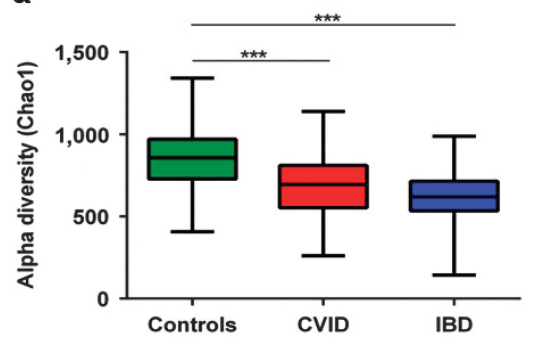

b

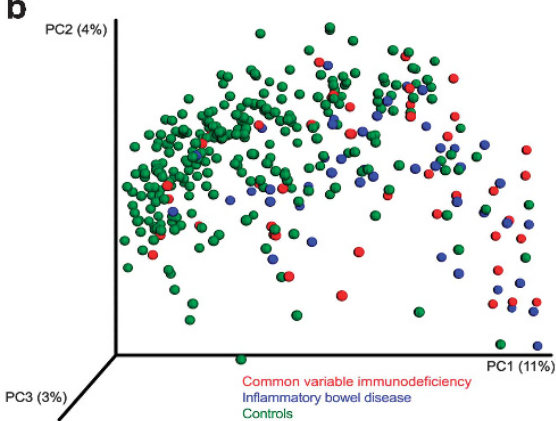

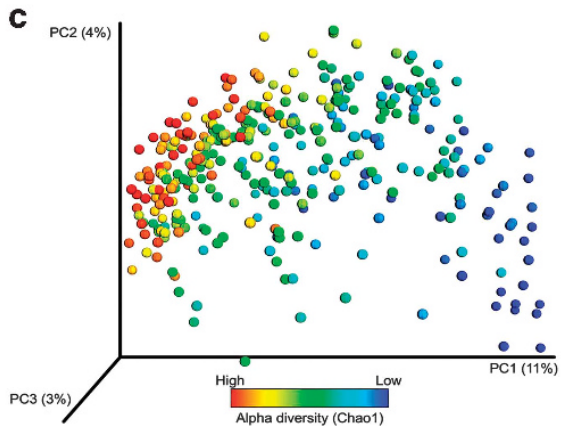

Figure 1 Low intra-individual microbial diversity in common variable immunodeficiency (CVID) patients. (a) Alpha diversity (Chao1) in controls and patients with CVID and inflammatory bowel disease (IBD) showed in median quartiles and range. The comparison is made by using $t$-test and significant $P$-value is marked as ${ }^{* \star *} P<0.001,{ }^{\star *} P<0.01,{ }^{\star} P<0.05$. (b) Beta diversity plot colored by disease phenotype (unweighted unifrac distance). A principal coordinate plots of the unweighted unifrac distance, where each point represents a single sample colored by phenotype. The distance between points represents how compositionally different the samples are from one another. PC1, PC2, and PC3 represent the three principal coordinates that capture most of the diversity (fraction of diversity captured shown in percent). (c) The same principal coordinate plot as in (b), but the samples are now colored according to the alpha diversity measure Chao1.

$(n=22)$, organ-specific autoimmunity $(n=12)$, autoimmunemediated cytopenia $(n=11)$, enteropathy $(n=13)$, granulomas $(n=4)$, or nodular regenerative hyperplasia of the liver $(n=2)$. All the CVID patients were on IgG substitution therapy (seven on intravenous Ig, 34 on subcutaneous Ig, and three on a combination of both). Plasma levels of LPS, sCD14, sCD25, and selected inflammatory cytokines (tumor necrosis factor (TNF), interleukin (IL)-6, IL-8, and IL-12) were analyzed in an expanded cohort of 104 CVID patients (mean age 46, 51\% males) and a separate control cohort of 30 healthy individuals (mean age 47, 53\% males), and are referred to as the plasma cohort. When referring to the CVID patients with both microbiota and plasma samples available, these are called the combined cohort $(n=40)$. The clinical characteristics of the cohorts are given in Supplementary Table S1 online.

\section{Lower diversity of the gut microbiota in CVID patients than in healthy controls}

Microbial alpha diversity (Chao1, a bacterial richness estimate reflecting the number of different taxa observed within each sample) was lower in CVID patients than in controls $(P<0.001$, Figure 1a). The difference remained significant $(P<0.001)$ after adjusting for parameters different between patients and controls (age, body mass index, and number of courses of antibiotics in the last year); (Table 1). The alpha diversity was similar in IBD and CVID patients; (Figure 1a).

Global differences in the gut microbiota were analyzed by measures of beta diversity (i.e., to which degree different taxa are shared between individuals or groups of individuals). CVID patients were different in beta diversity compared with controls (unweighted unifrac $P<0.001, R=0.45$; Figure 1b) and IBD $(P<0.001, R=0.08$; Figure 1b). As shown in Figure 1c, there was a strong relationship between microbial (alpha) diversity and beta diversity along principal coordinate 1, illustrating that the degree of different taxa shared between individuals (beta diversity) is dependent on the number of different taxa observed within each individual (alpha diversity), as previously observed in other phenotypes. ${ }^{23}$

\section{Different abundances of bacterial taxa in CVID patients and controls}

We found 25 taxa at different taxonomic levels that were significantly different in CVID patients compared with controls across three different statistical approaches (univariate Mann-Whitney $U$ test, LEfSe tool (https://huttenhower.sph. harvard.edu/galaxy/root), ${ }^{24}$ and MaAsLin, ${ }^{23}$ see Methods); (Table 2). According to phylogeny, these 25 taxa represented 10 different branches (Table 2 and Figure 2a). Four of the branches were more abundant in CVID compared with controls, including the classes Gammaproteobacteria and Bacilli (defining these at the lowest possible taxonomic level), whereas six branches were less abundant in CVID, including the Bifidobacterium genus.

The relative abundance of the 10 main taxa differentiating CVID patients and controls were used to calculate a CVIDspecific dysbiosis index according to a previously defined method. $^{23}$ The dysbiosis index was by definition higher in CVID than in controls, but also significantly higher than in IBD patients (Figure 2b). The dysbiosis index was associated with the alpha diversity findings $\left(\rho=-0.60, P=7.9 \times 10^{-5}\right.$, Figure 2c) suggesting that the abundance of 10 taxa largely captures the dysbiosis in CVID.

No association with the use of antibiotics the previous year We found no relationship between the number of courses of antibiotics in the last year (not including the last month, as this was an exclusion criterion) and alpha diversity or the dysbiosis index in CVID patients (Supplementary Figure S1, A and B). In addition, no difference in alpha diversity or dysbiosis index between CVID patients who had not used antibiotics $(n=16)$ and those who had used $\geqslant 1$ (range $1-7$ ) courses of antibiotics during the last year $(n=28)$ (Supplementary Figure S1, C and $\mathbf{D})$ were identified.

\section{Altered microbiota in CVID is linked to the presence of noninfectious complications and IgA levels}

CVID patients in the "Complications" subgroup $(n=35)$ had reduced alpha diversity of the gut microbiota $(P=0.04$, 


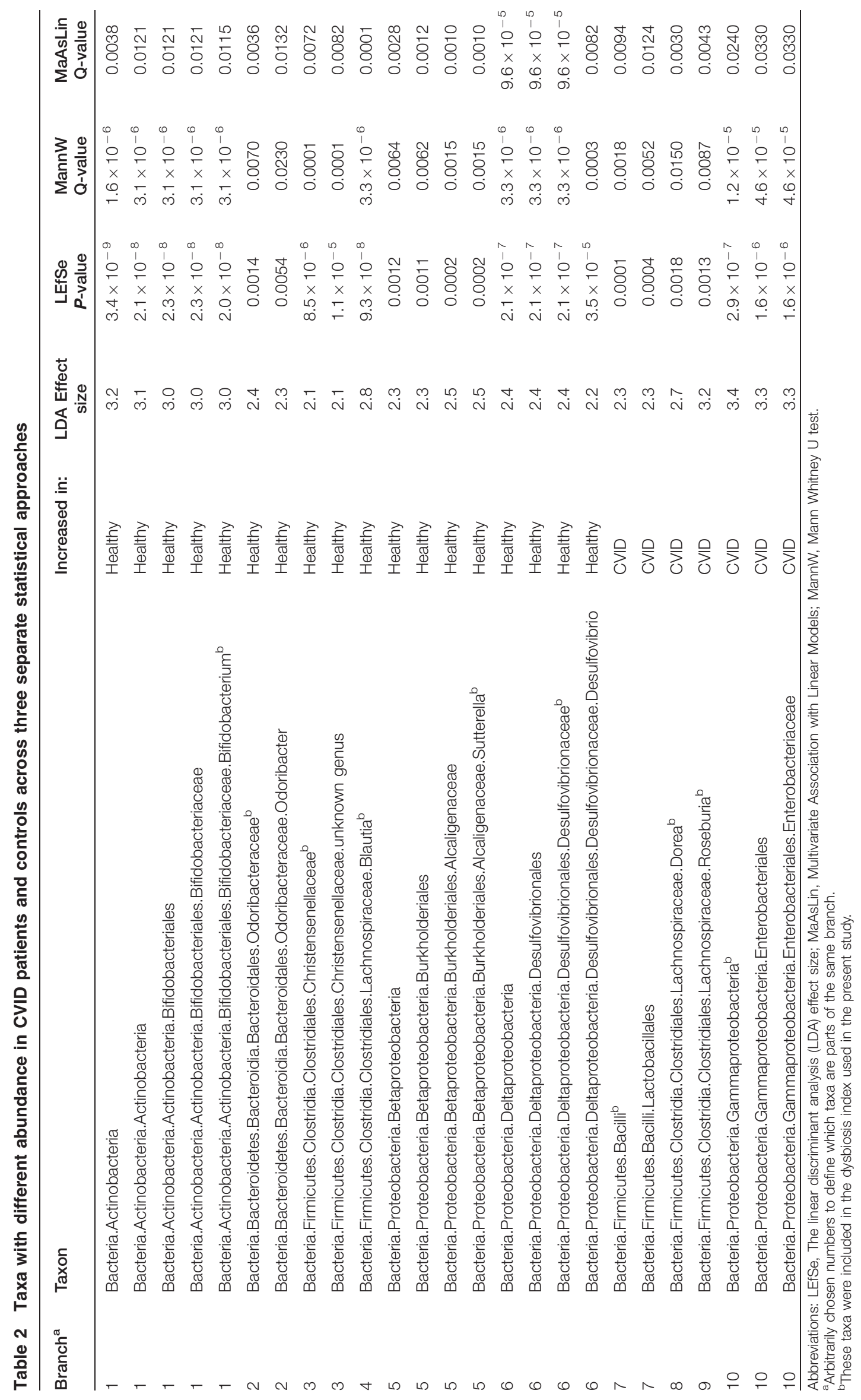



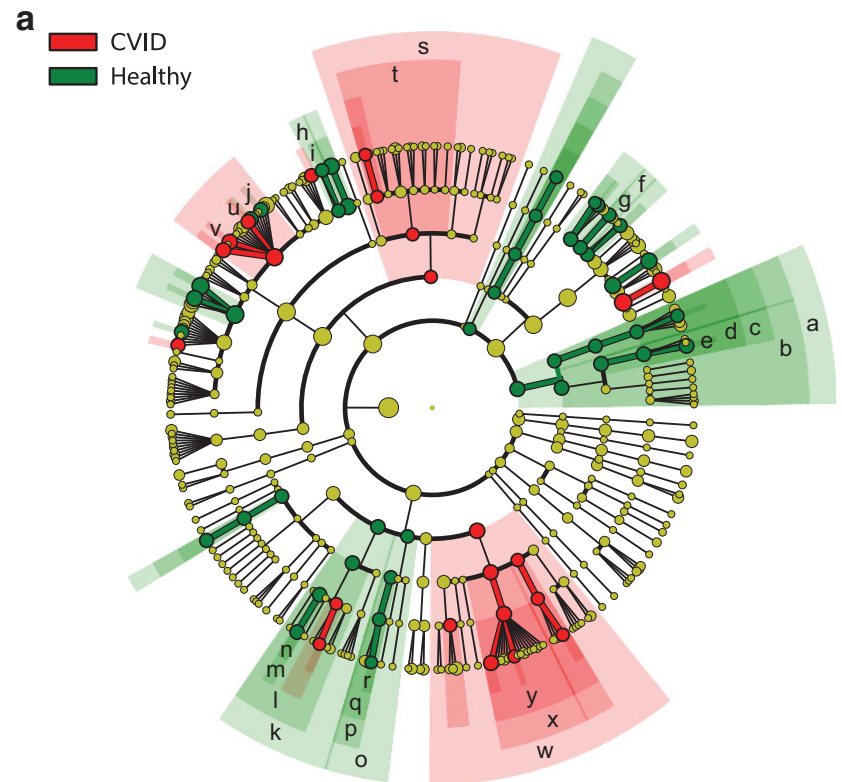

b

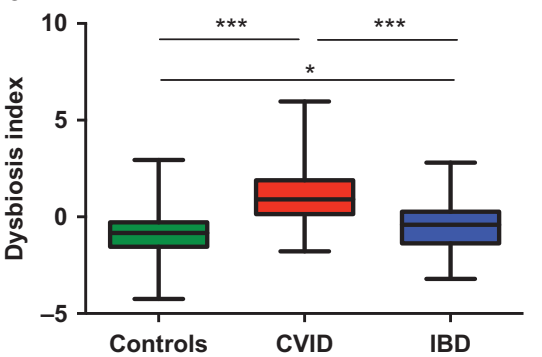

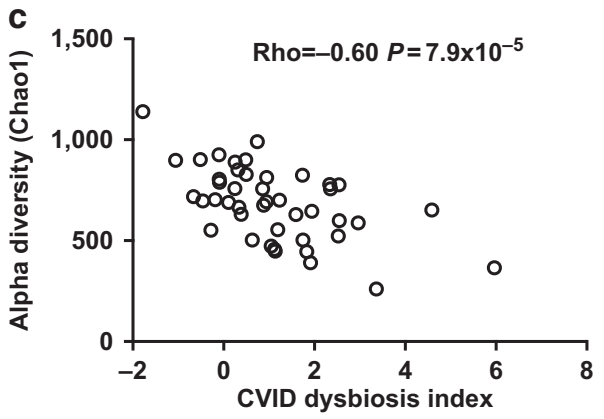

Figure 2 Dysbiosis index based on differentially distributed taxa. (a) A cladogram illustrating phylogenetic relationship between taxa, the center dot representing the kingdom Bacteria, the first circle representing Phylum, then the Class, Order, Family, and Genus levels. Taxa that are increased in common variable immunodeficiency (CVID) compared with controls are in red and taxa that are reduced in CVID compared with controls are in green. Named taxa are significant according to both univariate and multivariate statistics (Table 1) and are marked as small letters in the cladogram referring to corresponding taxa names in the legend at the right side of the figure. The vertical lines on the left side of the legend define taxa representing different levels of the same branch. The phylogenetic tree and coloring were made using LEfSe. ${ }^{24}$ (b) CVID-specific microbiota dysbiosis index (calculated from the abundances of the ten clades shown in (a)) applied in CVID, inflammatory bowel disease (IBD) and controls showed in median, quartiles, and range. The comparison is made by using Mann-Whitney $U$ test; significant $P$-value is marked as ${ }^{\star \star \star \star} P<0.001$, ${ }^{\star \star} P<0.01,{ }^{\star} P<0.05$. (c) Dysbiosis index correlates with alpha diversity (Chao1), Rho refers to Spearman's rank correlation coefficient.

Figure 3a) and increased CVID-specific dysbiosis index $(P=0.01$, Figure $3 \mathbf{b})$ compared with the "Infection only" subgroup $(n=9)$. When comparing with controls, the "Infection only" subgroup had elevated dysbiosis index but similar alpha diversity (Figure $\mathbf{3 a}$ and $\mathbf{c}$ ). There were no significant differences in the gut microbiota between the CVID patients with enteropathy within the "Complications" subgroup (Supplementary Table S2). Notably, the number of courses of antibiotics in both the CVID subgroups, "Infection only", and "Complications" were the same, suggesting an equal infection burden (median 2 infections per year, range $0-4.5$ and $0-7$, respectively, $P=0.91$ ). Also we found no statistical differences in the distribution of the Ig isotypes between these CVID subgroups (Supplementary Table S3).

The CVID patients were categorized according to their most recent Ig quantification (time between Ig quantification and stool samples was mean 2 months (range 0-11 months); (Supplementary Figure S2 and Supplementary Methods). Patients with very low serum $\operatorname{IgA}\left(\operatorname{IgA}<0.1 \mathrm{~g}^{-1}, n=35\right)$ had reduced alpha diversity compared with low to normal $\operatorname{Ig} \mathrm{A}\left(\operatorname{Ig} \mathrm{A} \geqslant 0.1 \mathrm{~g}^{-1}, n=9\right) ;(P=0.03$, Figure $3 \mathrm{c})$. However, the CVID-specific dysbiosis index was similar $(P=0.12)$. Importantly, the IgA level for each patient was reasonably stable in a 3-year-follow-up period after inclusion (Supplementary Table S4). In contrast to the association with IgA, there were no associations between the gut microbiota and the most recent $\operatorname{IgM}$ or $\operatorname{IgG}$ levels or B-cell phenotype (Supplementary Table S5). However, although $77 \%$ of the CVID patients were on weekly Ig subcutaneous infusion therapy giving a nearly constant serum level of IgG over time, the timespan between the stool sample and IgG quantification may have influenced the association analysis with alpha diversity and the dysbiosis index. 

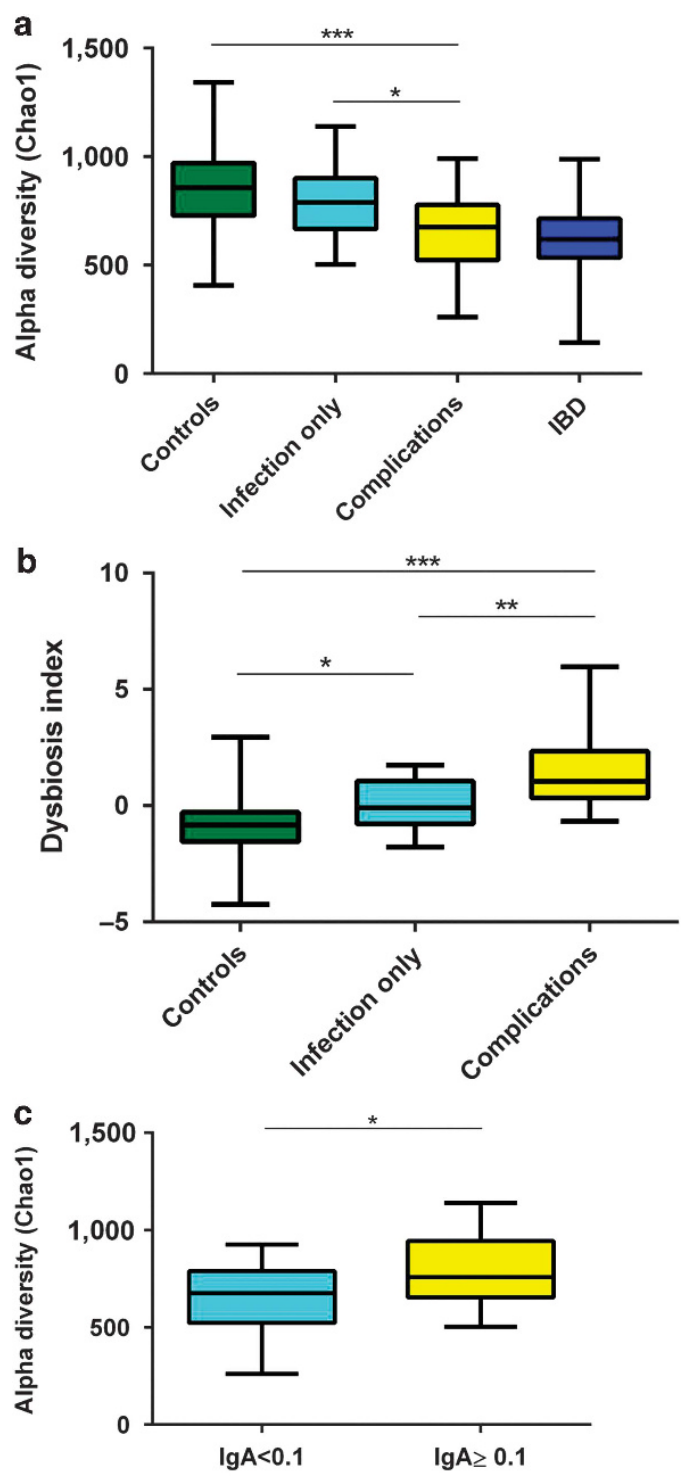

Figure 3 Altered gut microbiota associates with "Complications" subgroup and IgA levels in patients with common variable immunodeficiency (CVID). (a) Microbial alpha diversity (Chao1) in the "Infection only", "Complications", Controls, and inflammatory bowel disease (IBD) groups. (b) Dysbiosis index in the "Infection only", "Complications", and Controls. (c) Alpha diversity in the CVID patients with $\lg A<0.1 \mathrm{~g} / \mathrm{l}$ versus $\lg \mathrm{A} \geq 0.1 \mathrm{~g} / \mathrm{l}$. All the figures are shown in median, quartiles, and range. The comparisons are made by Mann-Whitney $U$ test in panel (a) and (b), and $t$-test in panel (c). Significant $P$-values are marked as ${ }^{* *} P<0.001,{ }^{\star *} P<0.01,{ }^{*} P<0.05$.

\section{Increased plasma levels of LPS associated with reduced} alpha diversity

Given the particular increase in CVID of the class Gammaproteobacteria that comprise many typical LPS-producing bacteria, we analyzed LPS levels in CVID patients in the plasma cohort $(n=104)$ compared with healthy controls $(n=30)$. We found increased LPS level in CVID patients compared with controls $(P=0.001$, Figure 4a). This difference was still significant $(P=0.001)$ after restricting the analysis to CVID patients in the combined cohort $(n=40$, time between plasma and stool samples was mean 4 months (range 0-9 months)).
Patients in the "Complications" subgroup had significantly higher LPS levels than the "Infection only" subgroup $(P=0.03$, Figure $4 \mathbf{b}$, plasma cohort). In the combined cohort, we found that in CVID patients, higher LPS levels were associated with lower alpha diversity $(\rho=-0.32, P=0.04$, Figure 4c), and higher dysbiosis index $(\rho=0.49, P=0.001$, Figure 4d). Of note, only Odoribacter (of the 25 taxa that were significantly different between CVID and healthy) was significantly associated with LPS $(\rho=-0.32, P=0.04$, Supplementary Table S6).

\section{Systemic T-cell activation associated with low alpha diversity}

To determine the level of systemic immune activation in CVID, we analyzed plasma levels of sCD14 (a marker of monocyte activation), sCD25 (i.e., soluble interleukin 2 receptor alpha, a marker of T-cell activation), and IL-6, IL-8, IL-12, and TNF (inflammatory cytokines reflecting $\mathrm{T}$-cell and macrophage activation) in the plasma cohort $(n=104)$. Plasma levels of sCD14, sCD25, as well as, IL-6, IL-8, IL-12, and TNF were all markedly elevated in patients with CVID compared with controls $(P<0.0001$ for all comparisons, Figure $5 \mathbf{a}$ and $\mathbf{b}$, Supplementary Table S7). When restricting the analysis to the combined cohort of CVID patients, higher SCD25 levels were associated with lower alpha diversity $(\rho=-0.40, P=0.01$, Figure 5c) and higher dysbiosis index $(\rho=0.42, P=0.007$, Figure 5d). In contrast, sCD14 were not significantly associated with either alpha diversity $(\rho=0.31, P=0.05)$ or higher dysbiosis index $(\rho=0.18, P=0.28)$. However, both sCD14 and sCD25 were markedly higher in the CVID subgroup "Complications" than in those with "Infection only" $(P=0.04$ and $P<0.0001$, respectively, Figure $5 \mathbf{e}$ and $\mathbf{f}$ ). None of the selected inflammatory cytokines (IL-6, IL-8, IL-12, and TNF) correlated with alpha diversity (Supplementary Table S8), potentially reflecting that the soluble CD25 antigen is a more stable and reliable marker of immune activation, which mirrors several up-stream inflammatory pathways than the selected cytokines that circulate at rather low levels. There was also an association in CVID patients between lower alpha diversity and lower percentage of naive $\mathrm{CD} 4{ }^{+} \mathrm{T}$ cells, a phenotypic characteristic of subgroups of CVID patients reported to be associated with a more severe phenotype ${ }^{25}(\rho=0.32, P<0.05$, Figure 5g). Mouillot et al. ${ }^{26}$ have suggested that CVID patients with naive $\mathrm{CD}^{+}{ }^{+} \mathrm{T}$ cells $<20 \%$ have more severe disease and we found that CVID patients with naive $\mathrm{CD} 4{ }^{+} \mathrm{T}$ cells $<20 \%$ had a significantly higher dysbiosis index (median $1.23(-0.09-4.59)$ ) than the other CVID patients (median 0.50( $-1.78-5.96)$ ), $P=0.024$.

We did not find a similar correlation between alpha diversity and sCD14 and sCD25 (or LPS), in IBD patients, although these results must be interpreted with caution as the analysis were performed on a smaller and separate IBD cohort $(n=16$, see Methods and Supplementary Figure S3).

\section{LPS and SCD25 independent predictors of alpha diversity and dysbiosis index}

We performed stepwise linear regression of parameters associated in the present study with alpha diversity (clinical 

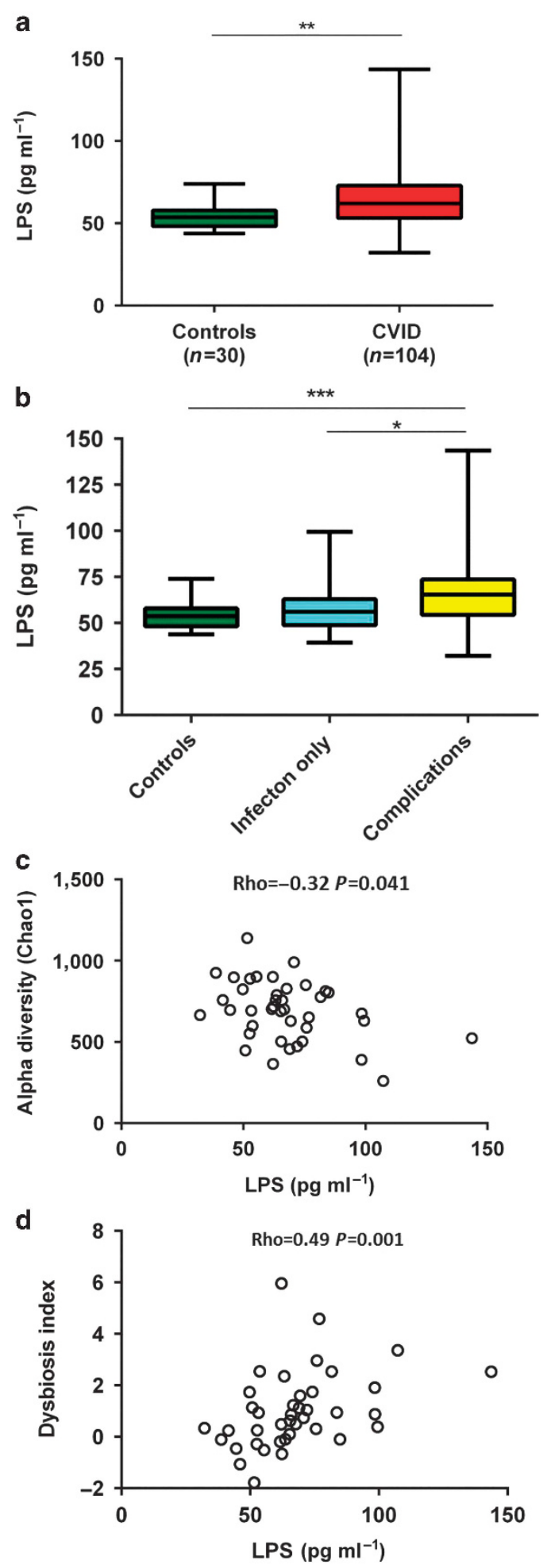

Figure 4 Increased lipopolysaccharide (LPS) in common variable immunodeficiency (CVID) patients correlates with altered gut microbiota. (a) LPS levels in an expanded CVID patient cohort $(n=104)$ compared with healty controls $(n=30)$ showed in median, quartiles, and range.

(b) LPS levels in "Infection only", "Complications", and Controls shown in median, quartiles, and range. (c) Alpha diversity Chao1 correlates with LPS. (d) Dysbiosis index correlates with LPS. The comparisons are made by Mann-Whitney $U$ test or Spearman's rank correlation test, as appropriate. Significant $P$-values are marked as ${ }^{\star \star \star} P<0.001,{ }^{* \star} P<0.01$, ${ }^{*} P<0.05$. Rho refers to Spearman's rank correlation coefficient. subgroup, LPS, sCD25, IgA, and \% of naive $\mathrm{CD} 4{ }^{+} \mathrm{T}$ cells) and dysbiosis index (clinical subgroup, LPS, sCD25). The levels of sCD25 and LPS were independent predictors of both alpha diversity (Beta: $-0.32, P=0.039$ and Beta: $-0.30, P=0.049$, respectively) and dysbiosis index (Beta: $0.40, P=0.007$ and Beta: $0.31, P=0.032$, respectively). LPS and $\mathrm{sCD} 25$ were not correlated $(\rho=0.02, P=0.86)$.

\section{DISCUSSION}

This is, to the best of our knowledge, the first study of the gut microbiota in a human primary immunodeficiency. The main findings were: (i) Large differences in the gut microbiota profile, both in terms of diversity and taxonomic profile, between CVID patients and controls without obvious associations to the use of antibiotics; (ii) Low alpha diversity in CVID patients was associated with elevated plasma levels of LPS and sCD25; (iii) CVID patients in the "Complications" subgroup exhibited the most extensive dysbiosis, elevated markers of immune activation, and LPS, highlighting this as a pathogenetic subgroup of CVID that differ from those with "Infection only". Our findings suggest a link between immunodeficiency, alterations of the gut microbiota, and systemic immune activation.

This study shows that CVID patients have extensive microbial dysbiosis. Dysbiosis has previously been detected in several metabolic disorders (e.g., obesity ${ }^{17}$ ), inflammatory diseases (e.g., $\mathrm{IBD}^{23}$ ), and also in relation to HIV infection. ${ }^{27}$ Using alpha diversity as a measure of the magnitude of the alterations, the difference in the microbial composition between CVID patients and controls was at the level of the disease control group IBD. However, as shown by the dysbiosis index, the taxonomic profile was significantly different in CVID and IBD. Notably, patients with CVID-related enteropathy defined as chronic diarrhea without infection did not differ from the other CVID patients in relation to microbiota-related parameters.

Mice models have shown that dysbiosis can be caused by changes in immunity ${ }^{28-30}$ and, reversely, that particular microbial species can elicit immunopathology in the presence of immunodeficiency. ${ }^{31,32}$ These examples illustrate that the interaction between the gut microbiota and the immune system is bidirectional, i.e., gut microbiota can induce alterations in the immune system and vice versa, the immune system can modulate the gut microbiota. Thus, without concluding on whether the dysbiosis is causing or caused by CVID, the current state of the gut microbiota may influence the host physiology and disease, and our findings may suggest that such mechanisms could be operating in CVID. Particularly, noteworthy differences between CVID patients and controls include the very low abundance of the genus Bifidobacterium in CVID patients, which is thought to have health-promoting properties and used in probiotic supplements. ${ }^{33}$ On the other hand, CVID patients were characterized by increase of the classes Bacilli and Gammaproteobacteria, harboring important pathogens in this patient group. 


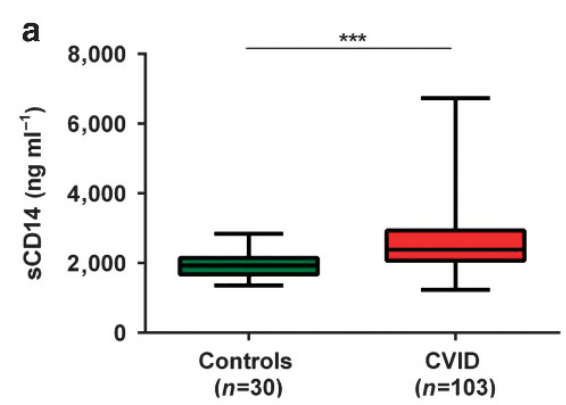

b
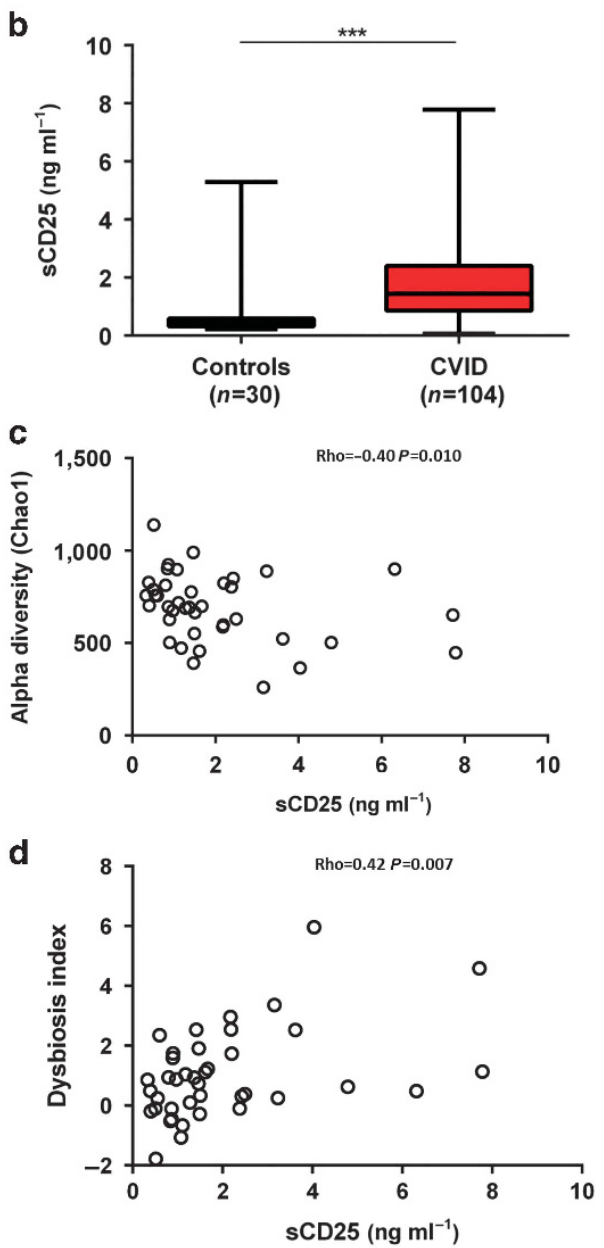

e

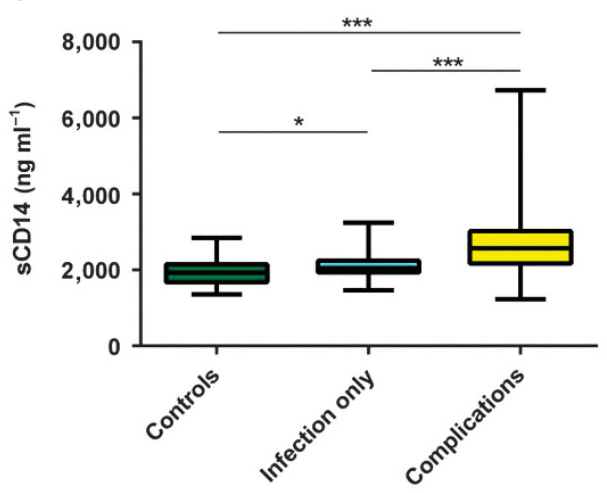

$\mathbf{f}$
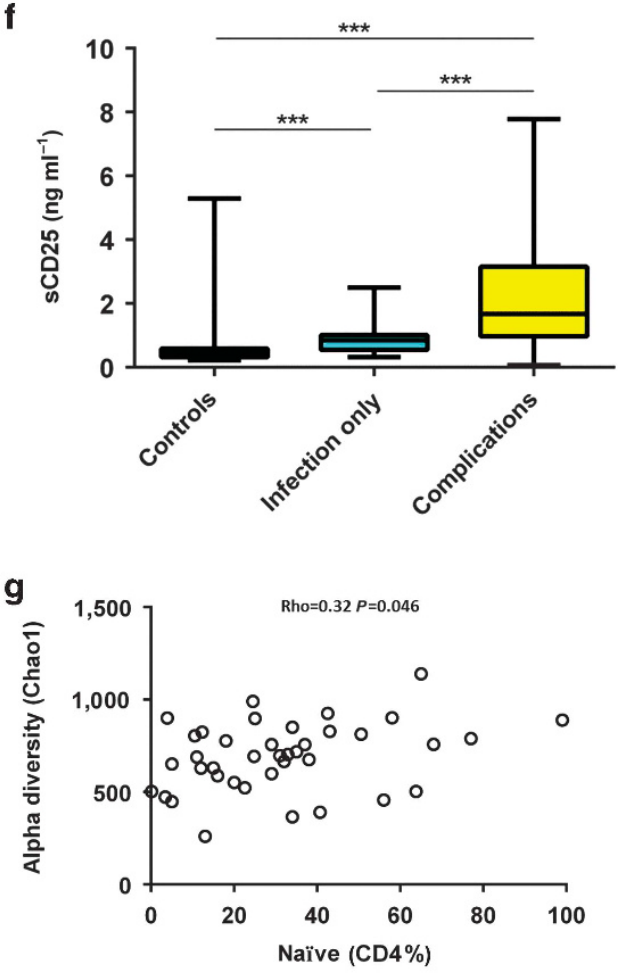

Figure 5 Elevated markers of monocyte and T-cell activation in CVID associates with alpha diversity and dysbiosis index. (a) Plasma levels of circulating soluble CD14 (sCD14) in CVID patients and controls showed in median, quartiles, and range. (b) Plasma levels of soluble CD25 (sCD25) in CVID patients and controls showed in median, quartiles, and range. (c) Correlation of SCD25 versus alpha diversity (Chao1) (d) Correlation of SCD25 versus dysbiosis index. (e) Plasma sCD14 in the "Infection only", "Complications", and Controls groups. (f) Plasma sCD25 in the "Infection only", "Complications", and Controls groups. (g) Correlation of \% naive CD4 and alpha diversity (Chao1). Comparisons are made by Mann-Whitney Utest or Spearman's rank correlation test, as appropriate. Significant $P$-value is marked as ${ }^{\star \star *} P<0.001,{ }^{\star \star} P<0.01,{ }^{\star} P<0.05$. Rho refers to Spearman's rank correlation coefficient.

LPS is a marker of microbial translocation that through interaction with toll-like receptor 4 is thought to be an important mediator of the chronic immune activation and inflammation seen in HIV-infected patients, ${ }^{34}$ as well as, in some cardiovascular disorders like chronic heart failure. ${ }^{35}$ In our study, plasma LPS levels were elevated in CVID patients and inversely correlated with the microbial alpha diversity and the degree of dysbiosis. It is also notable that
Bifidobacterium, which we found, reduced in CVID, was associated with significant improvement in gut barrier, lower intestinal, and plasma LPS levels, and reduced systemic inflammation in a mouse model of obesity. ${ }^{36}$ Overall, these findings suggest that the altered gut microbiota profile could possibly modulate the gut permeability with subsequent LPS elevation and chronic immune activation in CVID. ${ }^{16}$ 
In addition to LPS, high levels of SCD25, a reliable marker of $\mathrm{T}$-cell activation, were independently associated with alpha diversity and dysbiosis index in the CVID patients. Low levels of plasma IgA were also associated with reduced alpha diversity representing a parallel to the findings in a mouse model lacking IgA. ${ }^{22}$ LPS and sCD25 levels were however statistically stronger determinants of alpha diversity. A recent study in CVID patients suggested that LPS could contribute to T-cell dysfunction. ${ }^{21}$ However, in the present study $\mathrm{sCD} 25 \mathrm{did}$ not correlate with LPS suggesting that the interaction between the gut microbiota and systemic inflammation and immune activation could also involve mechanisms independent of LPS and toll-like receptors. We have also recently reported that alpha diversity correlates negatively with LPS and sCD14 in HIV-infected patients. ${ }^{37}$ Thus, associations between alpha diversity and markers of systemic immune activation are observed in both primary and acquired immunodeficiencies, and could reflect a more general phenomenon of the bidirectional interaction between the gut microbiota and the immune system.

Interestingly, reduced alpha diversity and increased LPS levels observed in CVID were only attributable to the subgroup with "Complications", whereas the alpha diversity and LPS levels in the "Infection only" subgroup were similar to the controls. These CVID subgroups have previously been shown to be different in relation to immunological and inflammatory markers as well as survival, ${ }^{5,6,38}$ and we are now able to demonstrate an altered gut microbial profile associating both with increased plasma levels of LPS and sCD25 as additional characteristics of this CVID subgroup.

CVID patients have a history of recurrent bacterial infections, and a critical question is therefore whether the phenotype under study is related to the use of antibiotics. Antibiotics have a substantial influence on the gut microbiota, and while the gut microbiota largely returns to the pretreatment state within 4 weeks after one course, ${ }^{39}$ the effect of repeated antibiotics courses is unpredictable. Although, in the present study there was no association between antibiotics in the last year and alpha diversity measures or dysbiosis index, accumulated use of antibiotics over years may have influenced the gut microbiota profiles of CVID patients, but probably in a nonsystematic way. ${ }^{40,41}$ The fact that the two subgroups (Infection only and Complications) are significantly different both in alpha diversity and dysbiosis index despite being exposed to a similar number of courses of antibiotics "in the last year", suggests that other factors than antibiotics are causing the higher dysbiosis index and lower alpha diversity in the "complication" subgroup, and thus CVID. Also, LPS levels in plasma were higher in the "complication" subgroup and not related to the use of antibiotics, suggesting that the LPS in these patients most probably reflect gut-leakage mechanisms and not ongoing infections, for example, in the respiratory tract. However, we cannot exclude that the long-term effects of antibiotics are more subtle than can be detected in a study with our sample size and depth of microbiota sequencing/ profiling.
Further studies on the effect of the "dysbiotic" CVID stool on intestinal inflammation in mice could establish whether it has an effect per se and not only is altered as a secondary phenomenon to CVID, e.g., by performing transplantation of fecal microbiota from CVID patients or controls into a relevant mouse model, as performed in obesity. ${ }^{42}$ In order to utilize the gut microbiota as a target for therapy in CVID, there is a need for studies aiming to explore if certain microbes or an altered gut microbiota can modulate systemic inflammation as well as the mechanisms for such immunomodulatory effects. This study provides a rationale for interventions targeting the gut microbiota, both to establish a causal relationship between the gut phenotype and CVID, as well as, to improve the therapeutic opportunities for this patient group.

\section{METHODS}

Study design. The CVID diagnosis was defined as decreased serum levels of IgG, IgA, and/or IgM by at least 2 standard deviations below the mean for age and exclusion of other causes of hypogammaglobulinemia. ${ }^{43,44}$ Clinical subgroup analysis were classified as "Infection only" or "Complications" based on previously defined criteria ${ }^{6}$ with one modification; CVID enteropathy was defined as persistent diarrhea after exclusion of gastrointestinal infection. Population controls $(n=263)$ were recruited from blood donors being part of the Norwegian Bone Marrow Donor Registry. The disease controls used for the gut microbiota analysis $(n=45)$, consisting of patients with Crohn's disease $(n=9)$, and ulcerative colitis $(n=36)$ were recruited from the outpatient clinic. In addition, the separate IBD cohort $(n=16)$ that had both gut microbiota and plasma analysis available were recruited from the gastroenterology outpatient clinic in Bergen, Norway (see Supplementary Figure 3).

The Regional Committee for Medical and Health Research ethics approved the study, and all participants signed informed consent.

Stool collection and analysis. All stool samples were collected by the participants at home with a standardized collection device ${ }^{45}$ and transferred to stool collection tubes with stool DNA stabilizer (Stratec Biomedical, Birkenfeld, Germany). ${ }^{46}$ The sample was returned by post together with a questionnaire reporting the sampling time, number of courses of antibiotics, weight, height, special diet, and medication. Upon arrival, the stool samples were immediately stored at $-20^{\circ} \mathrm{C}$, according to the manufacturer's recommendation, until DNA extraction. Bacterial DNA was extracted using the PSP Spin Stool DNA Plus Kit (Stratec Biomedical) and subjected to high-throughput sequencing of the $16 \mathrm{~S}$ ribosomal RNA gene with dual-indexed barcodes according to an established protocol. ${ }^{47}$ The final libraries were sequenced on an Illumina MiSeq (San Diego, CA; Supplementary Methods). For alpha diversity, Chao1, a bacterial richness estimate parameter was included in the analyses.

Blood sampling. Plasma for analyses of LPS, sCD14, sCD25, IL-6, IL-8, IL-12, and TNF were collected for 104 CVID patients and from 30 sex-and age-matched healthy controls (see Supplementary Methods). Serum levels of IgG, IgA, and IgM taken within 1 year of the stool sample and B cell ${ }^{48}$ (i.e., percentage of: CD19 ${ }^{+} \mathrm{B}$ cells, class switched B cells, CD $21^{\text {low }} \mathrm{B}$ cells, and transitional $\mathrm{B}$ cells) and $\mathrm{T}$ cell (i.e., percentage of naive and memory $\mathrm{CD} 4^{+}$and $\mathrm{CD} 8^{+} \mathrm{T}$ cells, and regulatory $\mathrm{T}$-cell $\left(\mathrm{CD} 4{ }^{+}, \mathrm{CD}_{4} 5 \mathrm{RO}^{+}, \mathrm{CD} 127^{\text {low }}, \mathrm{CD} 25^{+}\right)$) phenotypes assessed by flow cytometry (see Supplementary Methods), taken within one and a half years of the stool sample.

Statistical analysis. Univariate analyses were performed using parametric ( $t$-test) or non-parametric methods (Mann-Whitney $U$ and Kruskal-Wallis tests) for continuous variables, and $\chi^{2}$-test or Fisher's exact test for categorical variables, as appropriate. Correlation 
analysis was performed using parametric (Pearson) or non-parametric (Spearman) tests as appropriate, whereas linear regression was performed using stepwise forward method. Given the large number of taxa investigated, three different strategies were applied to identify taxa with particularly robust association to CVID: first univariate comparisons of the relative abundance of bacterial taxa at all taxonomic levels in CVID and controls were performed, including taxa with a false discovery rate according to the Benjamini-Hochberg method $\left(Q_{\mathrm{FDR}}\right)<0.05$ (Supplementary Table S9). Then we used the LEfSe tool, ${ }^{24}$ that applies univariate non-parametric statistics and linear discriminant analysis effect size ( $>2.0$ on a logarithmic scale) as exclusion criteria to identified taxa different in CVID and controls (Supplementary Table S9). Finally, MaAsLin ${ }^{23}$ that automatically selects and adjusts for confounding factors was used when identifying taxa that were significantly different between CVID and controls (with $\left.Q_{\mathrm{FDR}}<0.05\right)$, including age, gender, body mass index, library sequenced, and number of antibiotic courses last year as covariates (Supplementary Table S9). Only taxa that were significant across all three statistical approaches were presented in the Results section. For a full overview see Supplementary Table S9. For all other analyses, (alpha diversity, beta diversity, and dysbiosis index) uncorrected $P$-values are presented (unless stated otherwise) and $P<0.05$ were considered significant as the analyses were based on pre-specified hypothesis of subgroups and immune markers. Beta diversity (between group differences) was analyzed using ANOSIM test in QIIME (using 999 permutations). When not specified otherwise, statistical analyses were performed with SPSS (IBM, Armonk, NY) and R (cran.r-project.org).

CVID dysbiosis index. A CVID-specific microbial dysbiosis index was calculated according to Gevers et al. ${ }^{23}$ for all samples as $\log _{e}$ ((sum of the relative abundances of taxa upregulated in CVID)/(sum of the relative abundances of taxa reduced in CVID)), including (increased in CVID) Bacilli, Dorea, Roseburia, Gammaproteobacteria, and (reduced in CVID) Bifidobacterium, Odoribacteracea, Christensenellaceae, Blautia, Sutterella, Desulfovibrionacea. The most distant taxa in the phylogenic tree were chosen to be included in the dysbiosis index, with a few exceptions; if other taxa in the same tree had a higher LDA effect size and/or lower $P$-value, these were preferred.

SUPPLEMENTARY MATERIAL is linked to the online version of the paper at http://www.nature.com/mi

\section{ACKNOWLEDGMENTS}

The Norwegian Bone Marrow Donor Registry is acknowledged for providing the control population. Mona Bjørnstad, Liv Wenche Thorbjørnsen, Kristian Alfsnes, and Hege Dahlen Sollid, and other members of Norwegian PSC Research Center are acknowledged for support on sample collection and logistics. We thank Fredrik Bäckhed for helpful discussions. We thank the Fougner-Hartmann Familiefond (Denmark) for funding of an Illumina MiSeq sequencer. SFJ was funded by a grant from the SouthEastern Norway Regional Health Authority (project number 2012063). The project was also supported by grant number 911802 from Western Norway Regional Health Authority. JRH was funded by the Norwegian PSC Research Center and the Norwegian Research Council (project number 240787/F20).

\section{AUTHOR CONTRIBUTIONS}

SFJ, BF, PA, MK, AR, CD, MV, MLH, BM, KEAL and JRH recruited subjects, obtained human samples and clinical data collection. SFJ, MT, MK, JAA, TEM, BH, AEM, LTO, RKB, TU, JRH analyzed data and SFJ, MK, KH, TU, JRH performed statistical analysis. PA, THK and JRH coordinated and supervised the project. SFJ, JRH, PA, THK drafted the manuscript. All authors revised the manuscript for critical content and approved the final version.

\section{DISCLOSURE}

The authors declare no conflict of interest.

\section{REFERENCES}

1. Yong, P.F., Thaventhiran, J.E. \& Grimbacher, B. "A rose is a rose is a rose," but CVID is Not CVID common variable immune deficiency (CVID), what do we know in 2011? Adv Immunol. 111, 47-107 (2011).

2. Aukrust, P., Muller, F. \& Froland, S.S. Elevated serum levels of interleukin-4 and interleukin- 6 in patients with common variable immunodeficiency (CVI) are associated with chronic immune activation and low numbers of CD4 + Iymphocytes. Clin. Immunol. Immunopathol. 70, 217-224 (1994).

3. Aukrust, P., Muller, F. \& Froland, S.S. Enhanced generation of reactive oxygen species in monocytes from patients with common variable immunodeficiency. Clin. Exp. Immunol. 97, 232-238 (1994).

4. Aukrust, P., Svardal, A.M., Muller, F., Lunden, B., Berge, R.K. \& Froland, S.S. Decreased levels of total and reduced glutathione in CD4+ lymphocytes in common variable immunodeficiency are associated with activation of the tumor necrosis factor system: possible immunopathogenic role of oxidative stress. Blood 86, 1383-1391 (1995).

5. Resnick, E.S., Moshier, E.L., Godbold, J.H. \& Cunningham-Rundles, C. Morbidity and mortality in common variable immune deficiency over 4 decades. Blood 119, 1650-1657 (2012).

6. Chapel, H., Lucas, M., Lee, M., Bjorkander, J., Webster, D. \& Grimbacher, B. Common variable immunodeficiency disorders: division into distinct clinical phenotypes. Blood 112, 277-286 (2008).

7. Chapel, H. \& Cunningham-Rundles, C. Update in understanding common variable immunodeficiency disorders (CVIDs) and the management of patients with these conditions. Br. J. Haematol. 145, 709-727 (2009).

8. Salzer, U., Warnatz, K. \& Peter, H.H. Common variable immunodeficiency an update. Arthritis Res. Ther. 14, 223 (2012).

9. Li, J., Jorgensen, S.F., Maggadottir, S.M., Bakay, M., Warnatz, K. \& Glessner, J. Association of CLEC16A with human common variable immunodeficiency disorder and role in murine B cells. Nat. Commun. $\mathbf{6}$, 6804 (2015).

10. Maggadottir, S.M., Li, J., Glessner, J.T., Li, Y.R., Wei, Z. \& Chang, X. Rare variants at $16 \mathrm{p} 11.2$ are associated with common variable immunodeficiency. J. Allergy Clin. Immunol. 135, 1569-1577 (2015).

11. Fagarasan, S., Kawamoto, S., Kanagawa, O. \& Suzuki, K. Adaptive immune regulation in the gut: Tcell-dependent and T cell-independent IgA synthesis. Annu. Rev. Immunol. 28, 243-273 (2010).

12. Geuking, M.B., Cahenzli, J., Lawson, M.A., Ng, D.C., Slack, E. \& Hapfelmeier, S. Intestinal bacterial colonization induces mutualistic regulatory T cell responses. Immunity 34, 794-806 (2011).

13. Hooper, L.V., Littman, D.R. \& Macpherson, A.J. Interactions between the microbiota and the immune system. Science 336, 1268-1273 (2012).

14. Sutherland, D.B. \& Fagarasan, S. IgA synthesis: a form of functional immune adaptation extending beyond gut. Curr. Opin. Immunol. 24, 261268 (2012).

15. Lorenz, R.G., Chaplin, D.D., McDonald, K.G., McDonough, J.S. \& Newberry, R.D. Isolated lymphoid follicle formation is inducible and dependent upon lymphotoxin-sufficient B lymphocytes, lymphotoxin $\beta$ receptor, and TNF receptor I function. J. Immunol. 170, 5475-5482 (2003).

16. Tremaroli, V. \& Backhed, F. Functional interactions between the gut microbiota and host metabolism. Nature 489, 242-249 (2012).

17. Ley, R.E., Turnbaugh, P.J., Klein, S. \& Gordon, J.I. Microbial ecology: human gut microbes associated with obesity. Nature 444, 1022-1023 (2006).

18. Vrieze, A., Van Nood, E., Holleman, F., Salojarvi, J., Kootte, R.S. \& Bartelsman, J.F. Transfer of intestinal microbiota from lean donors increases insulin sensitivity in individuals with metabolic syndrome. Gastroenterology 143, 913-6 e7 (2012).

19. de Vos, W.M. \& de Vos, E.A. Role of the intestinal microbiome in health and disease: from correlation to causation. Nutr. Rev. 70 (Suppl 1), S45-S56 (2012).

20. Shulzhenko, N., Morgun, A., Hsiao, W., Battle, M., Yao, M. \& Gavrilova, O. Crosstalk between B lymphocytes, microbiota and the intestinal epithelium governs immunity versus metabolism in the gut. Nat. Med. 17, 1585-1593 (2011).

21. Perreau, M., Vigano, S., Bellanger, F., Pellaton, C., Buss, G. \& Comte, D. Exhaustion of bacteria-specific CD4 T cells and microbial translocation in common variable immunodeficiency disorders. J. Exp. Med. 211, 20332045 (2014). 
22. Kawamoto, S., Maruya, M., Kato, L.M., Suda, W., Atarashi, K. \& Doi, Y. Foxp3(+) T Cells regulate immunoglobulin a selection and facilitate diversification of bacterial species responsible for immune homeostasis. Immunity 41, 152-165 (2014).

23. Gevers, D., Kugathasan, S., Denson Lee, A., Vázquez-Baeza, Y., Van Treuren, W. \& Ren, B. The treatment-naive microbiome in new-onset Crohn's disease. Cell. Host. Microbe. 15, 382-392 (2014).

24. Segata, N., Izard, J., Waldron, L., Gevers, D., Miropolsky, L. \& Garrett, W.S. Metagenomic biomarker discovery and explanation. Genome Biol. 12, R60 (2011).

25. Giovannetti, A., Pierdominici, M., Mazzetta, F., Marziali, M., Renzi, C. \& Mileo, A.M. Unravelling the complexity of $T$ cell abnormalities in ;common variable immunodeficiency. J. Immunol. 178, 3932-3943 (2007).

26. Mouillot, G., Carmagnat, M., Gerard, L., Garnier, J.L., Fieschi, C. \& Vince, N. B-Cell and T-Cell phenotypes in CVID patients correlate with the clinical phenotype of the disease. J. Clin. Immunol. 30, 746-755 (2010).

27. Vujkovic-Cvijin, I., Dunham, R.M., Iwai, S., Maher, M.C., Albright, R.G. \& Broadhurst, M.J. Dysbiosis of the gut microbiota is associated with HIV disease progression and tryptophan catabolism. Sci. Transl. Med. 5, 193ra91 (2013).

28. Garrett, W.S., Lord, G.M., Punit, S., Lugo-Villarino, G., Mazmanian, S.K. \& Ito, S. Communicable ulcerative colitis induced by T-bet deficiency in the innate immune system. Cell 131, 33-45 (2007).

29. Elinav, E., Strowig, T., Kau, A.L., Henao-Mejia, J., Thaiss, C.A. \& Booth, C.J. NLRP6 inflammasome regulates colonic microbial ecology and risk for colitis. Cell 145, 745-757 (2011).

30. Vijay-Kumar, M., Aitken, J.D., Carvalho, F.A., Cullender, T.C., Mwangi, S. \& Srinivasan, S. Metabolic syndrome and altered gut microbiota in mice lacking Toll-like receptor 5. Science 328, 228-231 (2010).

31. Croker, B.A., Lawson, B.R., Rutschmann, S., Berger, M., Eidenschenk, C. \& Blasius, A.L. Inflammation and autoimmunity caused by a SHP1 mutation depend on IL-1, MyD88, and a microbial trigger. Proc. Natl Acad. Sci. USA 105, 15028-15033 (2008).

32. Henao-Mejia, J., Elinav, E., Jin, C., Hao, L., Mehal, W.Z. \& Strowig, T. Inflammasome-mediated dysbiosis regulates progression of NAFLD and obesity. Nature 482, 179-185 (2012).

33. Ventura, M., Turroni, F., Lugli, G.A. \& van Sinderen, D. Bifidobacteria and humans: our special friends, from ecological to genomics perspectives. J. Sci. Food Agric. 94, 163-168 (2014).

34. Brenchley, J.M., Price, D.A., Schacker, T.W., Asher, T.E., Silvestri, G. \& Rao, S. Microbial translocation is a cause of systemic immune activation in chronic HIV infection. Nat. Med. 12, 1365-1371 (2006).

35. Sandek, A., Bjarnason, I., Volk, H.D., Crane, R., Meddings, J.B. \& Niebauer, J. Studies on bacterial endotoxin and intestinal absorption function in patients with chronic heart failure. Int. J. Cardiol. 157, 80-85 (2012).

36. Cani, P.D., Possemiers, S., Van de Wiele, T., Guiot, Y., Everard, A. \& Rottier, O. Changes in gut microbiota control inflammation in obese mice through a mechanism involving GLP-2-driven improvement of gut permeability. Gut 58, 1091-1103 (2009).

37. Nowak, P., Troseid, M., Avershina, E., Barqasho, B., Neogi, U. \& Holm, K. Gut microbiota diversity predicts immune status in HIV-1 infection. AIDS 29, 2409-2418 (2015).

38. Cunningham-Rundles, C. The many faces of common variable immunodeficiency. Hematology 2012, 301-305 (2012).

39. Dethlefsen, L., Huse, S., Sogin, M.L. \& Relman, D.A. The pervasive effects of an antibiotic on the human gut microbiota, as revealed by deep $16 \mathrm{~S}$ rRNA sequencing. PLoS Biol. 6, e280 (2008).

40. Palmer, C., Bik, E.M., DiGiulio, D.B., Relman, D.A. \& Brown, P.O. Development of the human infant intestinal microbiota. PLoS Biol. 5, e177 (2007).

41. Koenig, J.E., Spor, A., Scalfone, N., Fricker, A.D., Stombaugh, J. \& Knight, R. Succession of microbial consortia in the developing infant gut microbiome. Proc. Natl Acad. Sci. USA 108 (Suppl 1), 4578-4585 (2011).

42. Ridaura, V.K., Faith, J.J., Rey, F.E., Cheng, J., Duncan, A.E. \& Kau, A.L. Gut microbiota from twins discordant for obesity modulate metabolism in mice. Science 341, 1241214 (2013).

43. Primary immunodeficiency diseases. Report of an IUIS Scientific Committee. International Union of Immunological Societies. Clin. Exp. Immunol. 118 Suppl 1, 1-28 (1999).

44. Al-Herz, W., Bousfiha, A., Casanova, J.L., Chatila, T., Conley, M.E. \& Cunningham-Rundles, C. Primary immunodeficiency diseases: an update on the classification from the international union of immunological societies expert committee for primary immunodeficiency. Front. Immunol. 5, 162 (2014).

45. Ahlquist, D.A., Schwartz, S., Isaacson, J. \& Ellefson, M. A stool collection device: the first step in occult blood testing. Ann. Intern. Med. 108, 609612 (1988).

46. Wu, G.D., Lewis, J.D., Hoffmann, C., Chen, Y.Y., Knight, R. \& Bittinger, K. Sampling and pyrosequencing methods for characterizing bacterial communities in the human gut using $16 \mathrm{~S}$ sequence tags. BMC Microbiol. 10, 206 (2010).

47. Kozich, J.J., Westcott, S.L., Baxter, N.T., Highlander, S.K. \& Schloss, P.D. Development of a dual-index sequencing strategy and curation pipeline for analyzing amplicon sequence data on the MiSeq Illumina sequencing platform. Appl. Environ. Microbiol. 79, 5112-5120 (2013).

48. Wehr, C., Kivioja, T., Schmitt, C., Ferry, B., Witte, T. \& Eren, E. The EUROclass trial: defining subgroups in common variable immunodeficiency. Blood 111, 77-85 (2008). 